\title{
Avaliação do peso e do modo de transporte do material escolar em alunos do ensino fundamental
}

\author{
Assessment of weight and mode of transport of school material in highschool students
}

Cláudia Tarragô Candotti ${ }^{1}$, Matias Noll², Eliane Roth ${ }^{3}$

\section{RESUMO}

Objetivos: Identificar e comparar o modo de transporte e o peso do material escolar utilizado por escolares entre o $2^{\circ}, 5^{\circ}$ e $9^{\circ}$ anos do ensino fundamental.

Métodos: Dentre as escolas do município Dois Irmãos (RS), uma escola foi sorteada e 58 escolares foram pesquisados por meio de questionário, que avaliou o acessório utilizado para transportar o material escolar e a forma de transporte. As variáveis antropométricas e o peso do material escolar foram mensurados ao longo de cinco dias. A análise do questionário envolveu tabelas de frequência e teste do qui-quadrado. $\mathrm{O}$ peso médio do material, relativo ao peso corporal e registrado ao longo da semana, foi analisado por ANOVA one-way e ao teste post hoc de Bonferroni.

Resultados: Escolares do $2^{\circ}, 5^{\circ}$ e $9^{\circ}$ anos transportam seu material nas costas, em mochila com duas alças (60, $77,3$ e $85,5 \%$, respectivamente, $p<0,05)$, apoiadas sobre os ombros $(60,77,3$ e $81 \%$, respectivamente; $p<0,05)$. A média do peso da mochila relativo ao peso corporal no $2^{\circ}$, $5^{\circ}$ e $9^{\circ}$ anos foi $7,2 \pm 2,3,8,8 \pm 3,0$ e $5,9 \pm 1,7 \%$, respectivamente, com diferença significativa apenas entre escolares do $5^{\circ}$ e $9^{\circ}$ anos.

Conclusões: Os escolares do $2^{\circ}, 5^{\circ}$ e $9^{\circ}$ anos transportam preferencialmente seu material escolar utilizando a mochila com duas alças nas costas apoiadas simetricamente sobre os ombros. Independentemente do ano escolar, o peso da mochila transportada foi inferior a $10 \%$ do peso corporal; os escolares do $5^{\circ}$ ano foram os que transportaram mochilas com maiores cargas.

Palavras-chave: postura; ensino; promoção da saúde; suporte de carga.

\section{ABSTRACT}

Objectives: To identify the way school material is carried and the weight of the material carried, and to compare these findings for $2^{\text {nd }}, 5^{\text {th }}$ and $9^{\text {th }}$ graders.

Methods: This study evaluated 58 students from a randomly selected school in Dois Irmãos, a city in southern Brazil, using anthropometric variables and a questionnaire to identify what schoolchildren used to carry school material and the way it was carried. School material was weighed over a five-day period. Descriptive statistics was used to analyze the responses to the questionnaires. Mean weight of the school material, normalized by body weight, was analyzed using one-way ANOVA and the Bonferroni post hoc test $(\square=0.05)$.

Results: Second, $5^{\text {th }}$ and $9^{\text {th }}$ graders carried their own material using a backpack with two straps $(60.0 \%, 77.3 \%$, and $85.5 \%, p<0.05)$ over their shoulders $(60.0 \%, 77.3 \%$, and $81.0 \%, p<0.05)$, and mean rate of schoolbag weight to body weight was $7.2 \% \pm 2.3,8.8 \% \pm 3.0$, and $5.9 \% \pm 1.7$. The only significant difference $(p<0.05)$ was between $5^{\text {th }}$ and $9^{\text {th }}$ graders.
Instituição: Universidade Federal do Rio Grande do Sul (UFRGS), Porto Alegre, RS, Brasil

'Doutora em Ciências do Movimento Humano pelo Programa de Pós-graduação em Ciências do Movimento da Escola de Educação Física da UFRGS; Professora do curso de Fisioterapia e do Programa de Pós-graduação da UFRGS, Porto Alegre, RS, Brasil

'Graduada em Educação Física pela Universidade do Vale do Rio dos Sinos, Porto Alegre, RS, Brasil

${ }^{3}$ Mestrando em Ciências do Movimento Humano pelo Programa de Pósgraduação em Ciências do Movimento Humano da Escola de Educação Física da UFRGS, Porto Alegre, RS, Brasil
Endereço para correspondência:

Cláudia Tarragô Candotti

Rua Fernando Osório 1.887 - Teresópolis

CEP 91720-330 - Porto Alegre/RS

E-mail: claudia.candotti@ufrgs.br

Conflito de interesse: nada a declarar

Recebido em: 24/1/2011

Aprovado em: 2/8/2011 
Conclusion: Students in the $2^{\text {nd }}, 5^{\text {th }}$ and $9^{\text {th }}$ grades prefer to carry their school material using a backpack with two straps symmetrically placed over the shoulders. Regardless of school grade, the backpack weight was less than $10 \%$ of body weight, and $5^{\text {th }}$ graders carried the heaviest relative loads.

Key-words: posture; teaching; health promoting; weight-bearing.

\section{Introdução}

A ocorrência de dor e de problemas posturais está disseminada na população adulta ${ }^{(1-4)}$, manifestando-se também, em grandes proporções, na infância e na adolescência ${ }^{(5-8)}$. Paananen $e a^{(9)}$ demonstraram que a ocorrência de dor musculoesquelética em duas ou mais áreas anatômicas é muito frequente em jovens escolares; Detsch et al ${ }^{(10)}$ verificaram prevalência de $66 \%$ para as alterações posturais laterais e de $70 \%$ para as alterações anteroposteriores da coluna vertebral na infância e adolescência.

Siivola $e t$ a $l^{(11)}$ demonstraram que a dor nas costas e os problemas posturais em jovens podem ter causa multifatorial. Utilizar mochilas pesadas e transportá-las de modo assimétrico, permanecer longos períodos de tempo em postura inadequada durante a posição sentada ${ }^{(11)}$, utilizar mobílias inadequadas, assistir por muito tempo à televisão ${ }^{(12)}$, realizar diferentes atividades de vida diária (AVD) com postura inadequada ${ }^{(13,14)}$, entre outros, são identificados como fatores de risco para o surgimento da dor nas costas e dos problemas posturais em jovens.

Visto que tanto a postura corporal mantida durante a atividade de transporte do material escolar quanto a magnitude da carga transportada são fatores de risco associados à dor nas costas e à ocorrência de problemas posturais ${ }^{(15-18)}$, especula-se que o transporte do material escolar tenha importantes implicações para a saúde e o bem-estar dos escolares, pois determina a quantidade e a distribuição do esforço sobre as estruturas musculoesqueléticas, podendo potencializar ou amenizar os malefícios e sobrecargas resultantes na coluna vertebral ${ }^{(3,19)}$.

Assim, sabendo-se que as alterações posturais e a dor nas costas estão associadas à falta de hábitos posturais adequados durante as $\mathrm{AVD}^{(1,20,21)}$, é relevante que estas variáveis sejam investigadas com profundidade na infância e juventude. Neste contexto, o estudo teve por objetivos: 1) identificar o modo de transporte e o peso do material escolar transportado e 2) comparar o modo de transporte e o peso do material transportado por escolares entre o $2^{\circ}$, $5^{\circ}$ e $9^{\circ}$ anos do ensino fundamental (EF).

\section{Método}

Trata-se de estudo transversal, com coleta prospectiva de dados, realizado no município de Dois Irmãos (RS), no período de junho a julho de 2010. Para definir o tamanho da amostra foi realizado um cálculo amostral com base na estimativa da média populacional de acordo com Santos, Abbud e $\mathrm{Abreu}^{(22)}$. Foi utilizado um grau de confiança de 95\%, erro máximo de estimativa de $10 \%$ sobre a média $(11,7 \%)$ do peso da mochila relativo ao peso corporal de escolares ${ }^{(19)}$ e desvio padrão $( \pm 4,3 \%)$ proveniente da literatura ${ }^{(19)}$. Desse modo, foi determinado um número mínimo de 52 escolares para que se pudesse cumprir com os propósitos do presente estudo.

Considerando que o ensino fundamental inicia-se no $1^{\circ}$ ano escolar e termina no $9^{\circ}$, totalizando nove anos escolares, no presente estudo, estes nove anos escolares foram classificados em três etapas: 1) anos iniciais, do $1^{\circ}$ ao $3^{\circ}$ ano; 2) anos intermediários, do $4^{\circ}$ ao $6^{\circ}$ ano; 3) anos finais, do $7^{\circ}$ ao $9^{\circ}$ ano.

Na primeira fase dos procedimentos de amostragem, após a definição da classificação dos anos escolares, foi realizado um sorteio simples ${ }^{(23)}$ entre todas as escolas de ensino fundamental completo (que possuíssem todos os nove anos escolares) existentes no município de Dois Irmãos, no Rio Grande do Sul, para eleger qual seria a escola participante do estudo.

$\mathrm{Na}$ segunda fase dos procedimentos de amostragem, depois de sorteada a escola participante, outro sorteio foi realizado, somente nesta escola, com objetivo de escolher quais turmas participariam do estudo ${ }^{(23)}$. Nesta fase, foi utilizada a classificação dos anos escolares, ou seja, para cada uma das etapas (anos iniciais, anos intermediários e anos finais) foi realizado um sorteio simples entre todas as turmas existentes na escola que se enquadravam na respectiva etapa. Sendo assim, foram sorteadas três turmas, $2^{\circ}, 5^{\circ}$ e $9^{\circ}$ ano do $\mathrm{EF}$, representando turmas iniciantes, intermediárias e em conclusão do EF, respectivamente.

Os critérios de inclusão foram: estar matriculados no EF da escola avaliada, ter entre sete e 18 anos de idade, não possuir lesão e/ou outro motivo que impedisse a criança/adolescente de levar a mochila escolar. Foram excluídos os escolares que faltaram em alguma das cinco aulas da semana em que foram realizadas as avaliações. 
Participaram 58 escolares de ambos os sexos, com idades entre sete e 16 anos, que estudavam no $2^{\circ}(n=15)$, $5^{\circ}(\mathrm{n}=22)$ e $9^{\circ}(\mathrm{n}=21)$ anos do EF desta escola. Todos os escolares participaram voluntariamente do estudo e tal participação foi autorizada pelos pais e/ou responsáveis, os quais assinaram o termo de consentimento livre e esclarecido. Da mesma forma, obteve-se a autorização da direção da escola para a realização do estudo em suas dependências. O estudo foi aprovado no Comitê de Ética em Pesquisa da UFRGS.

Foram realizados três procedimentos de coleta de dados: 1) Questionário; 2) Mensuração de variáveis antropométricas; 3) Mensuração do peso do material escolar.

O questionário (disponível com a autora correspondente) consistiu em quatro perguntas do tipo múltipla escolha, sendo que as duas primeiras apresentavam alternativas ilustradas com fotografias para facilitar a interpretação pelos escolares. Tal instrumento procurou avaliar a forma como os escolares estavam habituados a transportar o seu material escolar e foi construído especialmente para o estudo a partir de uma observação prévia dos tipos de mochilas, bolsas e pastas utilizadas pelos escolares desta escola para transportar seus materiais escolares.

Para determinar a especificidade do questionário, ou seja, a qualidade das informações oriundas do mesmo, seguiram-se os procedimentos padrão descritos na literatura ${ }^{(23,24)}$. Segundo Thomas e Nelson ${ }^{(24)}$, "os critérios fundamentais para julgar a qualidade das medidas utilizadas na coleta de dados da pesquisa são validade e fidedignidade". A validade da medida, determinada pela validade de conteúdo do questionário, indica o grau no qual o instrumento mede o que se espera que ele supostamente deva medir, ou seja, a validade se refere à segurança da interpretação do respectivo instrumento. Sendo assim, neste estudo, a validade de conteúdo do questionário foi determinada a partir da análise de três especialistas na área de educação postural. Os resultados desta análise demonstraram que o instrumento era aplicável e específico para avaliar com precisão o que objetivava. Ainda, segundo Thomas e Nelson (24) "a fidedignidade é uma importante parte da validade, que diz respeito à consistência ou a possibilidade de repetição de uma medida" (p.201). Para verificar a fidedignidade do questionário, o mesmo foi submetido ao procedimento de teste e reteste, com intervalo de 15 dias entre eles, com um grupo de 15 escolares não participantes da amostra ${ }^{(23,24)}$. Os resultados desse procedimento foram submetidos a testes de correlação de Spearman e de Wilcoxon, os quais demonstraram que todas as correlações foram fortes $(r \geq 0,94)$, significantes $(p \leq 0,01)$ e que não existiu diferença $(p \geq 0,9)$ entre as respostas do teste e reteste. Os resultados dos procedimentos de validação de conteúdo e de fidedignidade indicaram que o questionário contava com questões claras, objetivas e adequadas a esta faixa etária, apresentando altos índices de fidedignidade ${ }^{(23,24)}$.

A mensuração da estatura foi realizada com uma trena métrica fixada na parede, com sensibilidade de $1 \mathrm{~mm}$. O aluno foi posicionado de costas à parede, mantendo um olhar horizontal e sem calçados. A medida foi realizada do vértex até a região plantar. Para a leitura, foi utilizado um dispositivo que garantiu a horizontalidade ao longo da medida, evitando erros de medida decorrentes da inclinação do dispositivo. A medida da estatura foi registrada em centímetros.

A mensuração da massa corporal dos escolares foi realizada utilizando-se uma balança digital (Britânia), com precisão de $100 \mathrm{~g}$. Para esta avaliação, foi solicitado que os escolares estivessem com os pés descalços e trajando roupas leves.

Para a mensuração da massa do material escolar, as mochilas, bolsas ou pastas foram avaliadas durante uma semana, de segunda a sexta-feira. Nesses cinco dias, a massa do material escolar de todas as crianças foi repetidamente mensurada, para que o valor médio pudesse representar a realidade dos escolares e para que fosse possível acompanhar a variação das cargas ao longo da semana.

Os dados provenientes da estatura e massa corporal permitiram calcular o índice de massa corporal (IMC). O peso corporal $\left(\mathrm{p}_{\mathrm{c}}\right)$ dos escolares foi obtido multiplicandose a massa corporal pela aceleração da gravidade, sendo registrado em Newton. O peso do material escolar $\left(\mathrm{p}_{\mathrm{m}}\right)$ foi obtido multiplicando-se a massa do material escolar pela aceleração da gravidade, sendo registrado em Newton. Para análise do peso do material escolar, foi calculada a média aritmética simples dos pesos registrados ao longo da semana de mensuração. Também foi calculado o índice de proporção (IP, em porcentagem) entre as variáveis peso corporal e peso da mochila utilizando-se a equação IP $=p_{\mathrm{m}} \times 100 / p_{\mathrm{c}}$ ], sendo $p_{\mathrm{m}}$ a média semanal do peso do material escolar em Newton e $p_{c}$ o peso corporal do escolar em Newton. Quanto maior o valor do IP, maior é o valor do peso da mochila em relação ao peso corporal do escolar. 
A análise estatística foi realizada no software SPSS 17.0. A normalidade dos dados (teste de Shapiro Wilk) e a homogeneidade das variâncias (teste de Levene) foram confirmadas. Para verificar se existia diferença significativa do $\mathrm{p}_{\mathrm{m}}$ entre os dias da semana e do IP entre os anos escolares, foi utilizado a ANOVA one-way e o teste post hoc de Bonferroni. As variáveis nominais (tipo de acessório, modo de transporte, transporte de material desnecessário e solicitação de esforço) foram analisadas a partir de tabelas de frequência. Ainda, foi utilizado o teste do qui-quadrado para verificar se existia diferença significativa: 1 ) entre as frequências das respostas para cada turma (análise intraturma) e 2) entre as frequências das respostas para o $2^{\circ}$, $5^{\circ}$ e $9^{\circ}$ anos (análise interturma). O nível de significância adotado foi 0,05 .

\section{Resultados}

A Tabela 1 apresenta os resultados de cada ano escolar $\left(2^{\circ}, 5^{\circ}\right.$ e $9^{\circ}$ anos $)$ para as variáveis idade, peso corporal, estatura e IMC.

A Tabela 2 apresenta os valores do peso do material escolar referentes à média semanal e de cada dia, separadamente. Os resultados do teste ANOVA One-way demonstraram diferenças significativas, em cada ano escolar, do peso do material escolar entre os dias da semana (Tabela 2). Especificamente, no $2^{\circ}$ ano escolar, verificou-se diferença entre quinta e sexta-feira. No $5^{\circ}$ ano escolar, as diferenças encontradas foram entre segunda e quarta-feira e entre a quarta e quinta-feira. No $9^{\circ}$ ano escolar, a diferença foi entre segunda e sexta-feira.

A média do IP para $2^{\circ}, 5^{\circ}$ e $9^{\circ}$ anos foi de $7,2 \pm 2,3,8,8 \pm 3,0$ e $5,9 \pm 1,7 \%$, respectivamente. Esses resultados indicam que, em média, os escolares utilizam material escolar (mochilas) com peso inferior a $10 \%$ do peso corporal e que os escolares do $5^{\circ}$ ano são os que transportam maior carga relativa. Do total de escolares de cada turma avaliada, 26,7, 31,8 e $0 \%$ para $2^{\circ}, 5^{\circ}$ e $9^{\circ}$ anos, respectivamente, apresentaram índices de proporção maiores que $10 \%$. Os resultados do teste ANOVA one-way demonstraram que o IP apresentou diferença significativa apenas entre os escolares do $5^{\circ}$ e $9^{\circ}$ anos $(p=0,001)$.

Ao analisar as respostas do questionário referentes ao acessório utilizado para transportar o material escolar e o modo de transporte deste acessório, observou-se que independentemente do ano escolar, a mochila com duas alças é o acessório mais utilizado pela maioria dos escolares, ou seja, $60,77,3$ e $85,7 \%$, para o $2^{\circ}, 5^{\circ}$ e $9^{\circ}$ anos respectivamente, sendo esta transportada pela maioria dos escolares com as duas alças da mochila colocadas nas costas sobre os ombros $\left(50,77,3\right.$ e $81 \%$, para $2^{\circ}$, $5^{\circ}$ e $9^{\circ}$ anos respectivamente) (Tabela 3 ). A comparação entre os três anos escolares das respostas do questionário referentes ao acessório utilizado para transportar o material escolar e o modo de transporte deste acessório demonstrou não haver diferenças significativas entre os anos escolares.

A Tabela 4 descreve a frequência de respostas do questionário referentes aos materiais desnecessários transportados para a escola, ou seja, que não foram solicitados naquele dia letivo e a avaliação da exigência de esforço referente à quantidade de materiais exigida diariamente para as aulas. Ao analisar as respostas do questionário referentes aos materiais desnecessários transportados para a escola, observou-se que os escolares do $5^{\circ}$ ano são os que menos transportam $(p=0,01)$ esse tipo de material, em relação ao $2^{\circ}$ e $9^{\circ}$ ano escolar. Os resultados encontrados sobre a avaliação da exigência de esforço referente à quantidade

Tabela 2 - Média e desvio padrão do peso do material escolar das turmas de $2^{\circ}, 5^{\circ}$ e $9^{\circ}$ anos, em cada dia da semana, e a média dos cinco dias da semana

\begin{tabular}{lccc}
\hline Dias & $\mathbf{2}^{\mathbf{0}}$ ano & $\mathbf{5}^{\mathbf{0}}$ ano & $\mathbf{9}^{\circ}$ ano \\
\hline Segunda-feira & $22 \pm 7$ & $31 \pm 12^{\mathrm{b}}$ & $30 \pm 10^{\mathrm{d}}$ \\
Terça-feira & $19 \pm 6$ & $33 \pm 12$ & $34 \pm 12$ \\
Quarta-feira & $17 \pm 6$ & $41 \pm 12^{\mathrm{b}, \mathrm{c}}$ & $36 \pm 12$ \\
Quinta-feira & $24 \pm 8^{\mathrm{a}}$ & $31 \pm 12^{\mathrm{c}}$ & $36 \pm 10$ \\
Sexta-feira & $16 \pm 7^{\mathrm{a}}$ & $32 \pm 8$ & $41 \pm 10^{\mathrm{d}}$ \\
\hline Média & $19,8 \pm 6$ & $33,3 \pm 10$ & $35,3 \pm 9$ \\
\hline
\end{tabular}

Diferença significativa do peso do material entre dois dias da semana: ${ }^{\mathrm{a}} p=0,038 ;{ }^{\mathrm{b}} p=0,036 ;{ }^{\mathrm{c}} p=0,033 ;{ }^{\mathrm{d}} \mathrm{p}=0,013$

Tabela 1 - Média e desvio padrão de idade, peso corporal, estatura e índice de massa corporal das turmas de $2^{\circ}, 5^{\circ}$ e $9^{\circ}$ anos

\begin{tabular}{lcccc}
\hline Turmas & Idade (anos) & Peso corporal $\mathbf{( N )}$ & Estatura $\mathbf{( c m})$ & IMC $\mathbf{( k g / \mathbf { m } ^ { 2 } )}$ \\
\hline $2^{\circ}$ ano $(\mathrm{n}=15)$ & $7,9 \pm 0,4$ & $275 \pm 56$ & $130,3 \pm 0,7$ & $16,27 \pm 1,9$ \\
$5^{\circ}$ ano $(\mathrm{n}=22)$ & $10,4 \pm 0,8$ & $393 \pm 85$ & $146,1 \pm 0,8$ & $18,43 \pm 2,7$ \\
$9^{\circ}$ ano $(\mathrm{n}=21)$ & $14,5 \pm 1,1$ & $614 \pm 122$ & $169,4 \pm 0,8$ & $21,49 \pm 3,5$ \\
\hline
\end{tabular}

$\mathrm{N}$ : Newton 
Tabela 3 - Frequência do tipo de acessório utilizado para transportar o material escolar e do modo de transporte utilizado pelos escolares de $2^{\circ}, 5^{\circ}$ e $9^{\circ}$ anos escolares; comparação entre as frequências das respostas para cada turma (análise intraturma)

\begin{tabular}{|c|c|c|c|c|c|c|}
\hline \multirow{2}{*}{ Acessório } & \multicolumn{2}{|c|}{$2^{\circ}$ ano } & \multicolumn{2}{|c|}{$5^{\circ}$ ano } & \multicolumn{2}{|c|}{$9^{\circ}$ ano } \\
\hline & Frequência & $\%$ & Frequência & $\%$ & Frequência & $\%$ \\
\hline Mochila rodinha & 2 & 13,3 & 0 & 0 & 0 & 0 \\
\hline Pasta com alça & 1 & 6,7 & 2 & 9,1 & 0 & 0 \\
\hline Mochila com duas alças & $9^{a}$ & 60,0 & $17^{\mathrm{b}}$ & 77,3 & $18^{b}$ & 85,7 \\
\hline Bolsa & 2 & 13,3 & 1 & 4,5 & 0 & 0 \\
\hline Mochila com uma alça & 1 & 6,7 & 2 & 9,1 & 3 & 14,3 \\
\hline Total & 15 & 100,0 & 22 & 100,0 & 21 & 100,0 \\
\hline \multicolumn{7}{|l|}{ Modo de transporte } \\
\hline Mochila com duas alças nas costas & $9^{c}$ & 50,0 & $17^{c}$ & 77,3 & $17^{c}$ & 81 \\
\hline Rodinhas no chão & 1 & 6,7 & 0 & 0 & 0 & 0 \\
\hline Mochila com uma alça nas costas & 1 & 6,7 & 2 & 9,1 & 3 & 14,3 \\
\hline Bolsa em um lado & 1 & 6,7 & 0 & 0 & 0 & 0 \\
\hline Pasta na mão & 1 & 6,7 & 2 & 9,1 & 0 & 0 \\
\hline Bolsa atravessada & 1 & 6,7 & 1 & 4,5 & 0 & 0 \\
\hline $\begin{array}{l}\text { Mochila com duas alças, usando } \\
\text { uma nas costas }\end{array}$ & 1 & 6,7 & 0 & 0 & 1 & 4,8 \\
\hline Total & 15 & 100,0 & 22 & 100,0 & 21 & 100,0 \\
\hline
\end{tabular}

Maior frequência observada em relação aos demais acessórios: ${ }^{a} p=0,005 ;{ }^{b} p=0,001$. Maior frequência observada em relação aos demais modos de transporte: ${ }^{c} p=0,001$

Tabela 4 - Frequência de materiais não solicitados transportados para a escola e avaliação da exigência de esforço referente à quantidade de materiais solicitados diariamente para as aulas; comparação entre as frequências das respostas para cada turma (análise intraturma)

\begin{tabular}{|c|c|c|c|c|c|c|}
\hline \multirow{2}{*}{$\begin{array}{l}\text { Transporte de material } \\
\text { desnecessário }\end{array}$} & \multicolumn{2}{|c|}{$2^{\circ}$ ano } & \multicolumn{2}{|c|}{$5^{\circ}$ ano } & \multicolumn{2}{|c|}{$9^{\circ}$ ano } \\
\hline & Frequência & $\%$ & Frequência & $\%$ & Frequência & $\%$ \\
\hline Sim & 8 & 53,3 & 5 & 22,7 & 13 & 61,9 \\
\hline Não & 7 & 46,7 & $17^{a}$ & 77,3 & 8 & 38,1 \\
\hline Total & 15 & 100,0 & 22 & 100,0 & 21 & 100,0 \\
\hline \multicolumn{7}{|l|}{ Exigência de esforço } \\
\hline Muito pouco & 0 & 0 & 1 & 4,5 & 1 & 4,8 \\
\hline Pouco & 3 & 20,0 & 3 & 13,6 & 0 & 0 \\
\hline Razoável & $9^{b}$ & 60,0 & $12^{\mathrm{c}}$ & 54,5 & $12^{\mathrm{d}}$ & 57,1 \\
\hline Muito & 2 & 13,3 & 6 & 27,3 & 5 & 23,8 \\
\hline Exagerado & 1 & 6,7 & 0 & 0 & 3 & 14,3 \\
\hline Total & 15 & 100,0 & 22 & 100,0 & 21 & 100,0 \\
\hline
\end{tabular}

${ }^{a}$ Menor frequência de transporte de materiais desnecessários $(p=0,017)$. Maior frequência em relação aos demais níveis de exigência: ${ }^{b} p=0,017$; ${ }^{c} p=0,005 ;{ }^{\mathrm{d}} p=0,004$

de materiais exigida diariamente para as aulas (Tabela 4) demonstraram que o nível de exigência mais referido pelos escolares foi "razoável” (60, 54,5 e $57,1 \%$, para $2^{\circ}, 5^{\circ}$ e $9^{\circ}$ anos respectivamente). A comparação das respostas do questionário sobre a avaliação da exigência de esforço referente à quantidade de materiais exigida diariamente para as aulas demonstrou não haver diferenças entre os três anos escolares.

\section{Discussão}

Os objetivos deste estudo foram identificar e comparar o modo de transporte e o peso do material escolar transportado por escolares do $2^{\circ}, 5^{\circ}$ e $9^{\circ}$ anos do EF do município de Dois Irmãos (RS). Os principais resultados indicaram que, independentemente do ano escolar, o modo de transporte mais utilizado pela maioria dos escolares é a mochila de duas 
alças nas costas (75,9\% do total de estudantes), apoiadas sobre os ombros. Resultados semelhantes foram encontrados por Aparicio et al ${ }^{(25)}$ que, ao avaliarem o modo de transporte da mochila escolar de 203 escolares da cidade de Salamanca (Espanha), verificaram que praticamente a totalidade dos avaliados utilizavam a mochila com duas alças.

Outros estudos têm demonstrado uma preferência à utilização da mochila com as duas alças sobre os ombros, porém com percentuais muito inferiores ${ }^{(10,17,20)}$, fato este que gera preocupação, visto que o transporte de material escolar em posturas inadequadas está associado à ocorrência de dor lombar e dos sintomas musculoesqueléticos em escolares ${ }^{(7,20)}$.

Ainda, conforme Whittfield, Legg e Hedderly ${ }^{(19)}$, a maneira mais adequada dos escolares transportarem o material escolar é carregar a mochila na altura do dorso, com as duas alças sobre os ombros, sendo estas muito bem ajustadas, sem folgas. As alças com folgas podem provocar sobrecarga na região dorsal e ocasionar hipercifose torácica e, como compensação, hiperlordose lombar e cervical. Corroborando estes achados, van Gent et $a l^{(17)}$, Korovessis et al ${ }^{(20)}$, e Brackley e Stevenson ${ }^{(26)}$ demonstram que a mochila deve ser carregada simetricamente sobre os dois ombros, distribuindo a sua carga uniformemente, caso contrário, podem resultar torques de inclinação prejudiciais na coluna vertebral, que ao longo dos anos podem levar ao surgimento da dor lombar e dos sintomas musculoesqueléticos.

Para Brackley e Stevenson ${ }^{(26)}$, com base em parâmetros fisiológicos, a carga ideal máxima das mochilas deve ser entre 10 e $20 \%$ do peso corporal do escolar, enquanto outros autores, de forma mais cautelosa, demonstram que a quantidade de carga transportada não deve exceder a $10 \%$ do peso corporal do escolar ${ }^{(19,27,28)}$. Neste sentido, os valores médios encontrados para cada ano escolar, no presente estudo, encontram-se dentro da normalidade estipulada pela literatura. Ainda, os dados obtidos referentes ao peso do material transportado demonstraram que os escolares do $9^{\circ}$ ano são os que transportam maior quantidade de peso para a escola, porém quando realizada a proporcionalidade em relação ao peso corporal, são os escolares do $5^{\circ}$ ano os que transportam maior peso relativo. Do total de escolares, $20 \%$ apresentaram índices de proporção maiores que $10 \%$. Entretanto, em todas as turmas, nenhum escolar apresentou índice de proporção acima de $15 \%$.

Estudos similares ${ }^{(17,19,29)}$ avaliaram escolares e seus resultados demonstraram que o peso da mochila aumenta significativamente com o aumento da idade, variando entre 5 e $29 \%$ do peso corporal, excedendo o limite aconselhável de $10 \%$.
Em países como Espanha, Grécia, Holanda e Nova Zelândia, entre outros, percebe-se haver maior preocupação em avaliar o excesso de peso, o tempo gasto e o modo de transporte do material escolar, visto que são encontradas inúmeras publicações referentes a estas questões. Considerando que resultados provenientes de outros países podem não condizer com a realidade brasileira, entende-se não ser ideal extrapolá-los para a população brasileira, bem como utilizá-los como base para a criação de políticas públicas e educacionais. Ainda, considerando a lacuna existente na literatura a respeito de informações a cerca do modo de transporte do material escolar nas diferentes regiões do Brasil, salienta-se a importância das informações aqui demonstradas, como um primeiro passo, tanto no desenvolvimento de estudos desta natureza em diferentes regiões do Brasil, com amostras maiores e que investiguem mais informações - como, por exemplo, o tempo de transporte deste material -, quanto no desenvolvimento e proposição de políticas públicas na área educacional e de saúde pública.

Uma opção interessante para atenuar os problemas relacionados com a má postura nas AVD, como transporte inadequado ${ }^{(11,30)}$, excesso de peso ${ }^{(11,30)}$ e tempo gasto transportando o material escolar ${ }^{(31)}$, é a realização de um Programa de Educação Postural (PEP) no ambiente escolar, que tem como objetivo principal ensinar as posturas adequadas nas AVD, evitando assim que posturas inadequadas possam provocar danos à coluna vertebral ${ }^{(32,33)}$. Neste sentido, a literatura tem referenciado que participantes de PEP, em diferentes faixas etárias, tendem a modificar positivamente sua postura durante as atividades da vida diária( ${ }^{(32-36)}$. Não obstante, para desenvolver este tipo de programas, são necessários subsídios acerca da realidade dos escolares, para que o seu conteúdo tenha coerência com as suas necessidades ${ }^{(22-34)}$. Deste modo, mais uma vez justifica-se a realização de estudos que busquem compreender os diversos aspectos que envolvem a postura dos escolares. Assim, os resultados aqui apresentados podem nortear um planejamento da disciplina de Educação Física e até mesmo da escola como um todo. No entanto, apesar destes resultados serem importantes para promover uma maior conscientização dos educadores, não podem ser transferidos para todas as regiões do Brasil, pois sabe-se que existem muitas diferenças, tanto genéticas, sociais, quanto culturais, entre os diversos estados do Brasil; entende-se esta como a principal limitação do presente estudo. Assim, conclui-se que os escolares do $2^{\circ}, 5^{\circ}$ e $9^{\circ}$ anos utilizaram a mochila, preferencialmente com duas alças nas costas, sendo estas apoiadas simetricamente sobre os ombros, com peso inferior a $10 \%$ do peso corporal. 


\section{Referências bibliográficas}

1. Candotti CT, Noll M, Cruz M. Prevalência de dor lombar e os desequilibrios musculares em manicures. Rev Arq Movimento 2010;6:125-40.

2. Alperovitch-Najenson D, Santo Y, Masharawi Y, Katz-Leurer M, Ushvaev D, Kalichman L. Low back pain among professional bus drivers: ergonomic and occupational-psychosocial risk factors. Isr Med Assoc J 2010;12:26-31.

3. Karahan A, Bayraktar N. Determination of the usage of body mechanics in clinical settings and the occurrence of low back pain in nurses. Int J Nurs Stud 2004:41:67-75.

4. Elders LA, Burdorf A. Prevalence, incidence, and recurrence of low back pain in scaffolders during a 3-year follow-up study. Spine (Phila Pa 1976) 2004;29:E101-6.

5. Correa A, Pereira J, Silva M. Avaliação dos desvios posturais em escolares: estudo preliminar. Fisioter Bras 2005;6:175-8.

6. Sato $\mathrm{T}$, Ito $\mathrm{T}$, Hirano $\mathrm{T}$, Morita $\mathrm{O}$, Kikuchi R, Endo $\mathrm{N}$ et al. Low back pain in childhood and adolescence: a cross-sectional study in Niigata City. Eur Spine J 2008;17:1441-7.

7. Skoffer B. Low back pain in 15- to 16-year-old children in relation to school furniture and carrying of the school bag. Spine (Phila Pa 1976) 2007;32:E713-7.

8. Gunzburg R, Balagué F, Nordin M, Szpalski M, Duyck D, Bull D et al. Low back pain in a population of school children. Eur Spine J 1999;8:439-43.

9. Paananen MV, Auvinen JP, Taimela SP, Tammelin TH, Kantomaa MT, Ebeling HE et al. Phychosocial, mechanical, and metabolic factors in adolescents' musculoskeletal pain in multiple locations: a cross-sectional study. Eur J Pain 2010;14:395-401.

10. Detsch C, Luz AM, Candotti CT, Oliveira DS, Lazaron F, Guimarães LK et al. $P$. Prevalence of postural changes in high school students in a city in southern Brazil. Rev Panam Salud Publica 2007;21:231-8.

11. Siivola SM, Levoska S, Latvala K, Hoskio E, Vanharanta H, KeinänenKiukaanniemi S. Predictive factors for neck and shoulder pain: a longitudinal study in young adults. Spine (Phila Pa 1976) 2004;29:1662-9.

12. Trevelyan FC, Legg SJ. Back pain in school children - Where to from here? Appl Ergon 2006;37:45-54.

13. Vanderthommen M, Defaweux M, Tomasella M, Crielaard JM. The gesture behavior of patients with low back pain during a back school program: preliminary analysis of an evaluation test. Ann Readapt Med Phys 1999;42:485-92.

14. Ribeiro CC, Conesa AG. Lower back pain: prevalence and preventive programs in childhood and adolescence. Rev Iberoam Fisioter Kinesiol 2008;11:32-8.

15. Martelli RC, Traebert J. Descriptive study of backbone postural changes in 10 to 16 year-oldschoolchildren. Tangará-SC, Brazil, 2004. Rev Bras Epidemiol 2006;9:87-93.

16. Méndez FJ, Gómez-Conesa A. Postural hygiene program to prevent low back pain. Spine (Phila Pa 1976) 2001;26:1280-6.

17. van Gent C, Dols JJ, de Rover CM, Hira Sing RA, de Vet HC. The weight of schoolbags and the occurrence of neck, shoulder, and back pain in young adolescents. Spine (Phila Pa 1976) 2003;28:916-21.
18. Mackie HW, Legg SJ, Beadle J, Hedderly DI. Comparison of four different backpacks intended for school use. Appl Ergon 2003;34:257-64.

19. Whittfield J, Legg SJ, Hedderly DI. Schoolbag weight and musculoskeletal symptoms in New Zealand secondary schools. Appl Ergon 2005;36:193-8.

20. Korovessis P, Koureas G, Zacharatos S, Papazisis Z. Backpacks, back pain, sagittal spinal curves and trunk alignment in adolescents: a logistic and multinomial logistic analysis. Spine (Phila Pa 1976) 2005;30:247-55.

21. Spence SM, Jensen GM, Shepard KF. Comparison of methods of teaching children proper lifting techniques. Phys Ther 1984;64:1055-61.

22. Santos GR, Abbud EL, Abreu AJ. Determination of the size of samples: an introduction for new researchers. Rev Cient Symposium 2007;5:59-65.

23. Gaya AC, editor. Ciências do movimento humano: introdução à metodologia da pesquisa. Porto Alegre: Artmed; 2008.

24. Thomas JR, Nelson JK. Medida das variáveis de pesquisa. In: Thomas Jr, Nelson JK, editors. Métodos de pesquisa em atividade física. Porto Alegre: Artmed; 2002.

25. Aparicio QE, Nogueras MA, Sedín LN, Alonso RA, Pedraz SL, Arenillas CJ. Influence of the kind daily school stage in the weight pupils rucksacks. Fisioterapia 2005;27:6-15.

26. Brackley HM, Stevenson JM. Are children's backpack weight limits enough? A critical review of the relevant literature. Spine (Phila Pa 1976) 2004;29:2184-90.

27. Fernandes SM, Casarotto RA, João SM. Effects of educational sessions on school backpack use among elementary school students. Rev Bras Fisioter 2008; 12:447-53

28. Limon S, Valinsky LJ, Ben-Shalom Y. Children at risk: risk factors for low back pain in the elementary school environment. Spine (Phila Pa 1976) 2004;29:697-702.

29. Kellis E, Emmanouilidou M. The effects of age and gender on the weight and use of schoolbags. Pediatr Phys Ther 2010;22:17-45.

30. Neuschwander TB, Cutrone J, Macias BR, Cutrone S, Chambers H, Hargens AR. The effect of backpacks on the lumbar spine in children: a standing magnetic resonance imaging study. Spine (Phila Pa 1976) 2010;35:83-8.

31. Negrini S, Carabalona R. Backpacks on! Schoolchildren's perceptions of load, associations with back pain and factors determining the load. Spine (Phila Pa 1976) 2002;27:187-95.

32. Candotti CT, Macedo CH, Noll M, Freitas K. Postural school: a methodology adapted for children. Rev Arq Movimento 2009;5:34-49.

33. Candotti CT, Nunes SE, Noll M, Freitas K, Macedo CH. Effects of a postural program for children and adolescents after eight months of practice. Rev Paul Pediatr 2011;29:577-83.

34. Rebolho MC, Casarotto RA, João SM. Strategies for teaching postural habits to children: comic strips vs. practical experience. Fisioter Pesq 2009;16:46-51.

35. Candotti CT, Macedo CH, Noll M, Freitas K. Escola de Postura: uma metodologia adaptada aos pubescentes. Rev Mackenzie Educ Fis Esporte 2010;9:91-100.

36. Cardon G, De Clercq DL, De Bourdeaudhuij I. Effects of back care education in elementary schoolchildren. Acta Paediatr 2000;89:1010-7. 